\title{
Formulation Optimization and Performance Evaluation of Papain and Clove Oil based Chemo-mechanical Caries Removing Gel
}

\author{
Captain Khushali, Kale Rupali*, Shirolkar Satish \\ Department of Pharmaceutics, Dr. D. Y. Patil Institute of Pharmaceutical Sciences and Research, Pimpri, Pune, Maharashtra, INDIA.
}

\begin{abstract}
Objective: To formulate and optimise stable caries removing gel using naturally extracted papain and clove, for non- invasive chemo-mechanical removal of dental caries. Methodology: Gel formulation was prepared using natural polymer such as pectin along with other excipients and optimized for desired level of papain activity and viscosity for effective application on carious tooth using $3^{2}$ factorial design. Optimized formulation was evaluated for its physicochemical properties, in-vitro antibacterial activity and ex-vivo chemo-mechanical performance. Results: FTIR and DSC studies confirmed compatibility of papain with other excipients. Formulation with ratio of 2.5: 2.5: 0.225 pectin : propylene glycol : triethanolamine was found to give stable gel with viscosity of $7800 \mathrm{cp}$, proteolytic activity of $91.64 \%$ with satisfactory in-vitro antibacterial activity and ex-vivo chemomechanical performance. Conclusion: Papain and clove containing chemo-mechanical
\end{abstract}

caries removing gel can serve as potential alternative to other invasive caries removing techniques which will also reduce chances of microbial infection at the site of application with symptomatic pain relief.

Key words: Clove, Chemomechanical removal, Dental caries, ex-vivo performance, Papain.

\section{Correspondence:}

Kale Rupali*, Dr. D. Y. Patil Institute of Pharmaceutical Sciences and Research, Pimpri, Pune, Maharashtra-411018, INDIA.

Phone: 9376927834

Email: rupalikale07@gmail.com

DOI: 10.5530/jyp.2018.10.7

\section{INTRODUCTION}

The world health organization (WHO) in 2016 has reported that dental caries affects $60-90 \%$ of population and $80 \%$ of them do not visit dentist due to fear of anaesthesia and unpleasant conventional drilling techniques of removal of dental caries.' Dental caries is an endogenous infectious disease involving destruction of mineralized tissue because of acids produced by activation of bacteria which leads to glycolysis of dietary carbohydrates. Starting from outer surface of tooth, infection penetrates to pulp cavity causing tooth decay.

Carious dentine is categorized on the basis of zone closest to pulp and zone closest to oral cavity. These are termed as "inner carious" or "affected" dentine and outer carious or infected dentine. Affected dentine is remineralisable since it has no or very few bacterial and dry intact collagen. Whereas, infected dentine with degraded collagen and high bacterial load is non-remineralisable following minimum intervention dentistry principles, it is important to preserve inner carious material by removing outer infected dentine during treatment of dental caries.

Traditionally, dental caries removal was done with the help of sharp edged instrument which causes deleterious thermal and pressure effect resulting into excessive loss of healthy dental tissue. So there is need to identify effective caries removing technique which will preserve healthy dental tissue for further re-mineralization along with antibacterial and analgesic and local anaesthetic effect. Chemo-mechanical caries removal (CMCR) is a technique targets infected tissues and help in preserving healthy dental tissues. Natural enzyme, papain extracted from plant carica papaya is an endogenous protein has proteolytic activity which cleaves collagen molecules affected in dental caries and digest of dead cells resulting in removal of infected dentine.

Papain is reported as safe, non-cytotoxic as well as bio-compatible with oral tissues having bactericidal and bacteriostatic effect. But low chemical stability of papain limits the use of papain in formulation. Clove oil is traditionally known for its analgesic, local anaesthetic effect as well as antimicrobial property and is used as simple remedy for toothache.

The aim of current study is to provide stable papain and clove oil combination containing gel which can provide an advantage of atraumatic chemo-chemical removal of dental caries along with analgesic and local anesthetic effect, with ease of application and enhancing the technique of caries removal by making it more compliant for patient as well as dentist.

\section{MATERIALS AND METHOD}

\section{Materials}

Papain was purchased from Rashi Biotech Pvt. Ltd. Clove oil was purchased from Oswal chemicals. Pectin was purchased from Loba Chemie and Hammersten type casein was purchased from Sigma Aldrich.

\section{Methods \\ Drug: Excipient Compatibility}

Potential interactions of papain and other excipients was done by FTIR spectrophotometry (Shimadzu) and Differential Scanning Calorimetry (DSC-61000; Mettler Toledo) for papain and physical mixture with excipients.

\section{Preparation of papain and clove gel}

For preparation of gel formulation, preservatives were dissolved in purified water at $70^{\circ} \mathrm{C}$, disodium hydrogen phosphate and potassium dihydrogen phosphate were dissolved in above solution after cooling it to room temperature. Papain was dissolved in resulting solution under stirring. Pectin was wetted with propylene glycol separately; clove oil was added into it and stirred by using magnetic stirrer followed by addition of tween 80 . Triethanolamine was used to adjust $\mathrm{pH}$ to 7 . This homogenous 
mixture was added to papain solution and stirred to get homogenous gel formulation. Purified water was used to make up the volume.

\section{Experimental Design}

A $3^{2}$ full factorial design was used for experimental design. Ratio of pectin: propylene glycol: triethanolamine and concentration of tween 80 were used as independent variables as shown in table I and viscosity and \% papain activity were taken as dependent response variables. Statistical analysis of formulations was done by experimental trial of 9 combinations. Design expert software (version 10) was used for mathematical and graphical optimization of results.

\section{Characterization of gel formulation Physicochemical evaluation}

All formulated gels were observed for their colour by visual observation, viscosity by using Brookfield's viscometer at 100rpm and $\mathrm{pH}$ by using calibrated $\mathrm{pH}$ meter.

\section{Proteolytic activity of papain}

Accurately weighed gel equivalent to $100 \mathrm{mg}$ of papain was dissolved in $100.0 \mathrm{ml}$ phosphate cysteine disodium ethylene diaminetra-acetate buffer. It was sonicated to dissolve completely. Suitable dilutions were made using phosphate cysteine disodium ethylene diaminetra-acetate buffer up to $10.0 \mathrm{ml}$. This solution was assayed in triplicate by performing proteolytic assay for papain. Concentration of papain was determined by equation generated using standard curve.

\section{Clove oil content}

Accurately weighed quantity of gel equivalent to $20 \mathrm{mg}$ of clove oil was dissolved in $100.0 \mathrm{ml}$ methanol. Suitable dilutions were made, and absorbance was measured at $281 \mathrm{~nm}$. Clove oil content was determined by equation generated using standard curve.

\section{In-vitro antimicrobial studu}

Antimicrobial activity of papain-clove gel was determined by using zone-inhibition method by using MacConkey agar using Staphylococus aureus and Escherichia coli as model organisms.

\section{Ex-vivo performance 5}

Ex-vivo performance of formulated gel was checked by using extracted carious tooth collected from Dr. D. Y Patil Dental College, Pimpri, Pune. Sufficient quantity of gel was applied on carious portion with the help of syringe and allowed to react for $5 \mathrm{~min}$. followed by scrapping of carious dentine gently using sterile spoon excavator. Fresh gel was reapplied if necessary and carious dentine was scraped using similar procedure, until complete cleaning of teeth. Time and quantity of formulated gel required for complete caries removal was recorded.

\section{RESULTS}

FTIR and DSC results of mixture of papain along with its excipients showed distinct individual peaks and endotherms respectively without any interaction.

Since preliminary studies suggested effect of concentration ratio of pectin:propylene glycol:triethanolamine and concentration of tween 80 on viscosity and \% activity of papain in formulation, experiment was designed by varying these factors at 3 levels and statistical optimization was done by applying $3^{2}$ full factorial design using Design expert software. Compositions used for design of experiment with respect to its coded values and response values are shown in table II. Since clove oil content was not affected by composition, it was not considered for optimization. ANOVA results suggested linear model significant for viscosity with model $\mathrm{p}$ value of $<0.0001$ and $2 \mathrm{~F} 1$ model significant for percent papain activity with model $\mathrm{p}$ value of $<0.0001$. Response surface plots showing effect of two independent variables on viscosity and \% papain activity are shown in Figure 1 (A) and (B) respectively.

\section{Effect on viscosity}

Mathematical relationship generated for viscosity is expressed as equation (1)

Viscosity $(Y 1)=+5122.22+4310.0{ }^{\star} A+940.0{ }^{\star} B$.

\section{Effect on \% papain activity}

Mathematical relationship generated for $\%$ activity is expressed as equation (2)

$\%$ papain activity $(Y 2)=+55.04+10.28^{\star} A-6.77^{\star} B-18.88^{\star} A B$

Interpretation of surface response plots and equations generated by factorial design was done to study effect of variable parameters on viscosity and $\%$ papain activity.

\section{In-vitro antimicrobial activity}

Zone of inhibition (ZOI) observed for formulated papain-clove gel against $\mathrm{E}$-coli and $\mathrm{S}$. aureus showed significant antibacterial activity against respective organisms.

\section{Ex-vivo performance}

Ex-vivo performance study in excavated teeth was found to show complete remval of caries using papain-clove gel. Process of removal of dental caries is shown in Figure 2. It was observed that caries gets softened after application of gel and can be completely removed after two application of gel with in 15-20 min.

\section{DISCUSSION}

In the current study papain and clove oil based chemo-mechanical caries removing gel was prepared and optimized using factorial design. Optimized gel was evaluated for various physicochemical parameters and in-vitro antibacterial efficacy and ex-vivo efficacy of the formulation.

In this study, gel formulation was prepared using pectin as polymer, propylene glycol and triethanolamine as viscosity modifier, tween 80 as surfactant and preservatives and buffer components. Pectin is water soluble and biodegradable polysaccharide which has ability to form protective layer around papain. It was used to enhance stability of papain in gel formulation. Various formulations of papain-clove gel were tried varying concentration of other excipients such as viscosity modifier and surfactant. Drug-excipient compatibility studies of papain and excipient was performed using FTIR and DSC which confirmed no significant interaction indicating its compatibility with each other. Formulation was further optimized for viscosity and \% papain activity using $3^{2}$ full factorial design and interaction of different excipients was studied.

In case of dental application, placement of gel on the carious teeth is very important to show its action and viscosity is an important factor for the same. Polynomial equation and surface response plot indicated increase in viscosity (Y1) with increasing concentration ratio of pectin: propylene glycol: triethanolamine as well as concentration of tween 80 . This effect is might be due to increase in concentration of pectin and tween 80 which acts as gelling agent increasing viscosity of formulation..$^{20}$

Whereas \% papain activity (Y2) which is important to show its efficacy to remove dental caries also was found to be increased with increase in concentration ratio of pectin: propylene glycol: triethanolamine which may be due to formation of protective layer of pectin around papain. But there was decrease in \% papain activity (Y2) with increase in tween 80 which might be due to emulsifying action of tween 80 leading to agglomeration which affects papain stability. A negative coefficient of polynomial equation generated for $\%$ papain activity (Y2) indicated antagonistic effect of both the factors. Increase in concentration of both factors resulted in decrease of papain activity which might be due to 


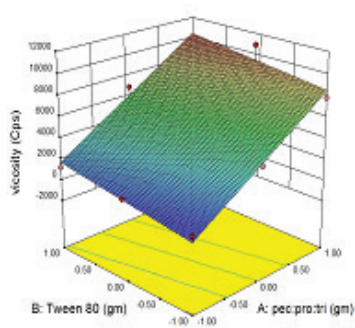

A

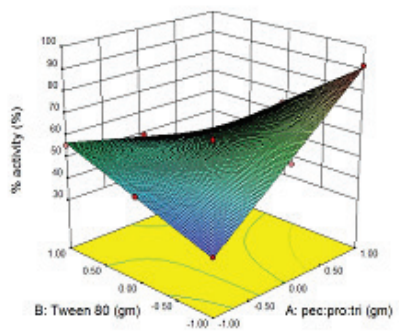

B

Figure 2: 3D surface response plots showing effect on A. Viscosity and B. $\%$ papain activity of formulation

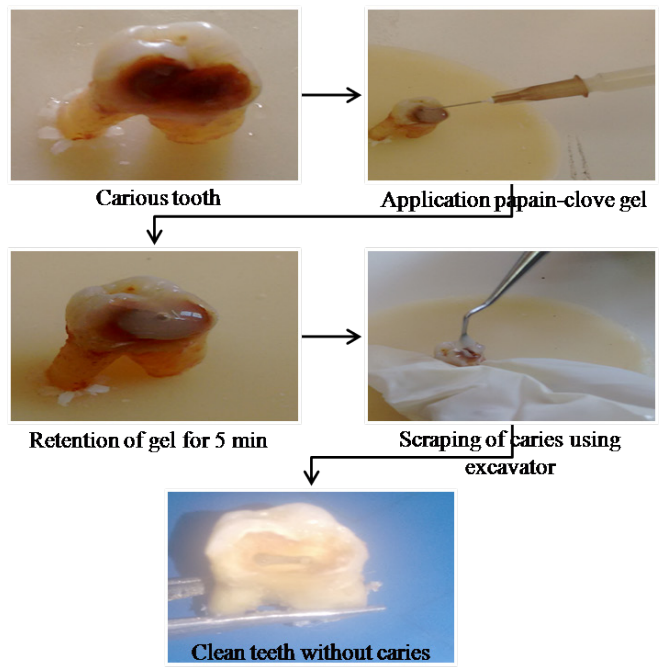

Figure 2: Ex-vivo efficacy of papain-clove gel on carious tooth

distruption of protective layer of pectin due to tween 80 as surface active agent. $^{21}$

Optimized formulation selected has $2.5 \mathrm{~g}$ papain, $0.5 \mathrm{~g}$ clove oil, $2.5 \mathrm{~g}$ pectin, $2.5 \mathrm{~g}$ propylene glycol, $0.125 \mathrm{~g}$ tween $80,0.0094 \mathrm{~g}$ potassium di-hydrogen phosphate, $0.032 \mathrm{~g}$ disodium hydrogen phosphate, $0.025 \mathrm{~g}$ methyl paraben, $0.0025 \mathrm{~g}$ propyl paraben which showed highest $\%$ activity of papain indicating its stability and viscosity of 7800cps due to which it can be easily retained on carious tooth cavity.

Efficacy of developed formulation against bacterial infection and caries removal were important parameters of evaluation. If the formulation cannot show antibacterial efficacy, it will lead to bacterial colonization and if caries cannot be removed completely secondary caries can be developed. Optimized gel formulation was evaluated for in-vitro antibacterial activity against $E$. coli and $S$. aureus which proved its antimicrobial efficacy.

Ex-vivo performance study of optimized papain and clove containing caries removing gel was found to remove carious dentine selectively without keeping any traces of caries behind. Caries became soft after application of prepared gel due to proteolytic action of papain. This softened mass can be easily removed by mild scrapping without causing any harm to healthy dentine. Presence of clove oil in the gel also may eliminate the use of anaesthetic for caries removal and its antibacterial action can give an additional antimicrobial protection.

\section{CONCLUSION}

Papain and clove oil containing gel formulation was developed and optimized based on its viscosity and stability of papain. In-vitro antimicrobial studies confirmed its antimicrobial efficiency and ex-vivo results indicated satisfactory performance for removal of dental caries. It can be used as suitable chemo-mechanical caries removal agent. It also may give additional advantages of analgesic and antibacterial activity other than proteolytic activity of papain due to presence of clove oil.

\section{CONFLICT OF INTEREST}

The authors declare no conflict of interest.

\section{REFERENCES}

1. Petersen PE, Ogawa $\mathrm{H}$. Prevention of dental caries through the use of fluoride -the WHO approach. Community Dental Health. 2016;33(2):66-8.

2. Banerjee A, Watson TF, Kidd EA. Dentine caries excavation: a review of current clinical techniques. Br Dent J. 2000;188(9):476-82.

3. DomenickTZ, Carloo GC, Margharita F. The biology, diagonosis and treatment of dental caries. J Am Dental Assoc. 2009;140:25-35.

4. Rajesh RH, Rao SN, Jobin J, Narayana CR, Moidin S, Shashidhar R. Evaluation of the efficacy of $2 \%$ curcumin gel in the treatment of experimental periodontitis. Pharmacognosy Research. 2014;6(4):326-33.

5. Beeley JA, Yip HK, Stevenson AG. Chemo-mechanical caries removal: a review of techniques and latest development. Br Dent J. 2000;188(8):427-30.

6. Ericson D, Zimmerman M, Raber $\mathrm{H}$. The efficacy of new gel as chemo mechanical caries removal agent. J Dent Res. 1998;77:1252-57.

7. Pushpalatha G, Sowmya S, Sanjay V, Santhosh KR, Melvin M, Sateesh CP, et al. Botulinum toxin-An Innovative Treatment Approach In Dental Practice. Journa of Young Pharmacists. 2016;8(1):2-5

8. Bussadori SK, Castro LC, Galvao AC. Papain gel: A new chemo mechanical caries removal agent. J Clin Pediatr Dent. 2005;30(2):115-9.

9. Banerjee A, Kidde AM, Watson TF. Scanning electron microscopic observations of human dentine after mechanical caries excavation. J Dent. 2000;28(3):179-86.

10. Bussadori SK, Guedes CC, Hermida BML, Ram D. Chemo-mechanical removal of caries in an adolescent patient using Papain gel: Case report. J Clin Pediatr Dent. 2008;32(3):177-80.

11. Pratap KM, Nandakumar K, Sambashivarao P, Sandhya PS. Chemo mechanical caries removal - a new horizon. Indian J Dent Adv. 2011;3(4):668-72.

12. Bhat SS, Sain S, Hegde SK. Efficacy of chemo-mechanical caries removal. IJADS. 2015;1(3):27-30.

13. Chakravarthy PK, Acharya S. Efficacy of extrinsic stain removal by nove dentifrice containing papain and bromelain extracts. J Young Pharmacists. 2012;4(4):245-9

14. Martins MD, Fernandes KP, Motta LJ, Santos EM, Pavesi VC Bussadori SK Biocompatibility analysis of chemo-mechanical of caries removal material Papacarie on cultured fibroblasts and subcutaneous tissue. J Dent Child. 2009; 76(2):123-9.

15. Osato JA, Santiago LA, Remo GM, Caudra MS, Mori A. Antimicrobial and antioxidant antioxidant activities of unripe papaya. Life Sci. 1999;53(17):1383-9.

16. Sankali MG, Mashru RC, Sankalia JM. Papain entrapment in alginate beads for stability improvement and site specific delivery: Physiochemical characterization and factorial optimization using neural network modelling. AAPS Pharm Sci Tech. 2005;6(2):E209-22.

17. Chellam M, Nadarajan S, Arun KD, Pujari SS. Chemical profiling of leaf essential oil, Antioxidant potential and Antibacterial activity of Syzygium lanceolatum (Lam.) Wt. \& Arn. (Myrtaceae). Free Rad Antiox. 2016;6:13-22.

18. Hosseini M, Asl MK, Rakhshandeh H. Analgesic effect of clove essential oil in mice AJP. 2011;1(1): 1-6

19. Kokare CR. Pharmaceutical microbiology Experiments and techniques, $2^{\text {nd }}$ ed Career Publications. 2008.136-40.

20. Sriamornsak P. Chemistry of pectin and its pharmaceutical uses: A Review. SUIJ. 2003;3(1-2):206-28.

21. Agarkhed M, Zhang J, Shrivastav A, Goldstein J, Dell C, Hsieh MC. Effect of polysorbate 80 concentration on thermal and photolytic stability of a monoclonal antibody. AAPS Pharm Sci Tech. 2013;14(1);1-9. 